\title{
STRONG K-STABILITY AND ASYMPTOTIC CHOW-STABILITY
}

\author{
TOSHIKI MABUCHI* AND YASUFUMI NITTA**
}

\begin{abstract}
For a polarized algebraic manifold $(X, L)$, let $T$ be an algebraic torus in the group $\operatorname{Aut}(X)$ of all holomorphic automorphisms of $X$. Then strong relative K-stability (cf. [6]) will be shown to imply asymptotic relative Chow-stability. In particular, by taking $T$ to be trivial, we see that asymptotic Chow-stability follows from strong K-stability.
\end{abstract}

\section{INTRODUCTION}

In this paper, we consider a polarized algebraic manifold $(X, L)$, i.e., a nonsingular irreducible projective variety $X$, defined over $\mathbb{C}$, with a very ample line bundle $L$ on $X$. Let $T$ be an algebraic torus in $\operatorname{Aut}(X)$. Then the main purpose of this paper is to show the following:

Main Theorem. If $(X, L)$ is strongly $K$-stable relative to $T$, then $(X, L)$ is asymptotically Chow-stable relative to $T$.

\section{Relative Chow-Stability}

For the maximal compact subgroup $T_{c}$ of $T$, we put $\mathfrak{t}_{c}:=\operatorname{Lie}\left(T_{c}\right)$. For every positive integer $\ell$, we consider the space $V_{\ell}:=H^{0}\left(X, L^{\otimes \ell}\right)$ endowed with a Hermitian metric $\rho_{\ell}$ such that the infinitesimal action of $\mathfrak{t}_{c}$ on $V_{\ell}$ preserves the metric $\rho_{\ell}$. Put $\mathfrak{t}:=\operatorname{Lie}(T)$ and $n:=\operatorname{dim} X$. Since the infinitesimal action of $\mathfrak{t}$ on $X$ lifts to an infinitesimal action of $\mathfrak{t}$ on $L$, we view $\mathfrak{t}$ as a Lie subalgebra, denoted by $\mathfrak{t}_{\ell}$, of $\mathfrak{s l}\left(V_{\ell}\right)$ by taking the traceless part. Let $\left(\mathfrak{t}_{\ell}\right)_{\mathbb{Z}}$ be the kernel of the exponential map

$$
\mathfrak{t}_{\ell} \ni y \mapsto \exp (2 \pi \sqrt{-1} y) \in \operatorname{SL}\left(V_{\ell}\right) .
$$

Let $\mathfrak{z}_{\ell}$ denote the centralizer of $\mathfrak{t}_{\ell}$ in $\mathfrak{s l}\left(V_{\ell}\right)$, and we consider a symmetric bilinear form $\langle,\rangle_{\ell}$ on $\mathfrak{s l}\left(V_{\ell}\right)$ defined by

$$
\langle u, v\rangle_{\ell}=\operatorname{Tr}(u v) / \ell^{n+2}, \quad u, v \in \mathfrak{s l}\left(V_{\ell}\right),
$$

2010 Mathematics Subject Classification. Primary 32Q26; Secondary 14L24, 53C25.

${ }^{*}$ Supported by JSPS Grant-in-Aid for Scientific Research (A) No. 20244005.

${ }^{* *}$ Supported by JSPS Grant-in-Aid for Young Scientiists (B) No. 23740063. 
whose asymptotic limit as $\ell \rightarrow \infty$ plays an important role (cf. [9]) in the study of relative K-stability for test configurations. We now consider the set $\mathfrak{t}_{\ell}^{\perp}$ of all $u \in \mathfrak{z} \ell$ such that

$$
\langle u, v\rangle_{\ell}=0 \quad \text { for all } v \in \mathfrak{t} .
$$

By the infinitesimal $\mathfrak{t}_{\ell}$-action on $V_{\ell}$, we can write the vector space $V_{\ell}$ as a direct sum of $\mathfrak{t}_{\ell}$-eigenspaces

$$
V_{\ell}=\bigoplus_{k=1}^{m_{\ell}} V\left(\chi_{\ell ; k}\right),
$$

for mutually distinct additive characters $\chi_{\ell ; k} \in \operatorname{Hom}\left(\left(\mathfrak{t}_{\ell}\right)_{\mathbb{Z}}, \mathbb{Z}\right), k=1,2, \ldots, m_{\ell}$, where $V\left(\chi_{\ell ; k}\right)$ denotes the space of all $\sigma \in V_{\ell}$ such that

$$
u \sigma=\chi_{\ell ; k}(u) \sigma \quad \text { for all } u \in\left(\mathfrak{t}_{\ell}\right)_{\mathbb{Z}} .
$$

Since $T_{c}$ acts isometrically on $\left(V_{\ell}, \rho_{\ell}\right)$, the subspaces $V\left(\chi_{\ell ; k}\right)$ and $V\left(\chi_{\ell ; k^{\prime}}\right)$ are orthogonal if $k \neq k^{\prime}$. For the Lie subalgebra $\mathfrak{s}_{\ell}$ of $\mathfrak{s l}\left(V_{\ell}\right)$ defined by

$$
\mathfrak{s}_{\ell}=\bigoplus_{k=1}^{m_{\ell}} \mathfrak{s l}\left(V\left(\chi_{\ell ; k}\right)\right),
$$

we consider the associated algebraic subgroup $S_{\ell}:=\Pi_{k=1}^{m_{\ell}} \operatorname{SL}\left(V\left(\chi_{\ell ; k}\right)\right)$ of $\mathrm{SL}\left(V_{\ell}\right)$. Let $Z\left(S_{\ell}\right)$ be the centralizer of $S_{\ell}$ in $\mathrm{SL}\left(V_{\ell}\right)$. Then the Lie algebra $\mathfrak{z} \ell$ is written as a direct sum of Lie subalgebras

$$
\mathfrak{z} \ell=\mathfrak{z}\left(\mathfrak{s}_{\ell}\right) \oplus \mathfrak{s} \ell
$$

where $\mathfrak{z}\left(\mathfrak{s}_{\ell}\right):=\operatorname{Lie}\left(Z\left(S_{\ell}\right)\right)$. For the Lie subalgebra $\mathfrak{t}_{\ell}^{\prime}:=\mathfrak{t}_{\ell}^{\perp} \cap \mathfrak{z}\left(\mathfrak{s}_{\ell}\right)$ of $\mathfrak{z}\left(\mathfrak{s}_{\ell}\right)$, we consider the associated algebraic subtorus $T_{\ell}^{\prime}$ of $Z\left(S_{\ell}\right)$. Then

$$
T_{\ell}^{\perp}:=T_{\ell}^{\prime} \cdot S_{\ell}
$$

is a reductive algebraic subgroup of $\mathrm{SL}\left(V_{\ell}\right)$ with the Lie algebra $\mathfrak{t}_{\ell}^{\perp}$. Let $\left(\mathfrak{t}_{\ell}^{\perp}\right)_{\mathbb{Z}}$ denote the set of all $u \in\left(\mathfrak{t}_{\ell}^{\perp}\right)_{\mathbb{Z}}$ in the kernel of the exponential map

$$
\mathfrak{z}_{\ell} \ni u \mapsto \exp (2 \pi \sqrt{-1} u) \in \mathrm{SL}\left(V_{\ell}\right)
$$

such that the circle group $\{\exp (2 \pi s \sqrt{-1} u) ; s \in \mathbb{R}\}$ acts isometrically on $\left(V_{\ell}, \rho_{\ell}\right)$. For each $u \in\left(\mathfrak{t}_{\ell}^{\perp}\right)_{\mathbb{Z}}$, by varying $s \in \mathbb{C}$, let

$$
\psi_{u}: \mathbb{G}_{m} \rightarrow \mathrm{SL}\left(V_{\ell}\right), \quad \exp (2 \pi s \sqrt{-1}) \mapsto \exp (2 \pi s \sqrt{-1} u),
$$

be the algebraic one-parameter group generated by $u$, where $\mathbb{G}_{m}$ denotes the 1-dimensional algebraic torus $\mathbb{C}^{*}$. Let $X_{\ell}$ be the image of $X$ under the Kodaira embedding

$$
\Phi_{\ell}: X \rightarrow \mathbb{P}^{*}\left(V_{\ell}\right)
$$


associated to the complete linear system $\left|L^{\otimes \ell}\right|$ on $X$. For the degree $d_{\ell}$ of $X_{\ell}$ in $\mathbb{P}^{*}\left(V_{\ell}\right)$, we put $W_{\ell}^{*}:=\left\{\operatorname{Sym}^{d_{\ell}}\left(V_{\ell}^{*}\right)\right\}^{\otimes n+1}$. Let $\hat{X}_{\ell} \in W_{\ell}^{*}$ be the Chow form for the irreducible reduced algebraic cycle $X_{\ell}$ on $\mathbb{P}^{*}\left(V_{\ell}\right)$, so that the associated point $\left[\hat{X}_{\ell}\right]$ in $\mathbb{P}^{*}\left(W_{\ell}\right)$ is the Chow point for $X_{\ell}$. Then the action of $T_{\ell}^{\perp}$ on $V_{\ell}$ induces an action of $T_{\ell}^{\perp}$ on $W_{\ell}^{*}$ and also on $\mathbb{P}^{*}\left(W_{\ell}\right)$.

Definition 2.1. (1) $\left(X, L^{\otimes \ell}\right)$ is called Chow-stable relative to $T$, if the following conditions are satisfied:

(a) The isotropy subgroup of $T_{\ell}^{\perp}$ at $\left[\hat{X}_{\ell}\right]$ is finite;

(b) The orbit $T_{\ell}^{\perp} \cdot \hat{X}_{\ell}$ in $W_{\ell}^{*}$ is closed.

(2) $(X, L)$ is called asymptotically Chow-stable relative to $T$, if there exists a positive integer $\ell_{0}$ such that $\left(X, L^{\otimes \ell}\right)$ are Chow-stable relative to $T$ for all positive integers $\ell$ satisfying $\ell \geq \ell_{0}$.

\section{Test COnfigurations}

Let $u \in\left(\mathfrak{t}_{\ell}^{\perp}\right)_{\mathbb{Z}}$. For the complex affine line $\mathbb{A}^{1}:=\{z \in \mathbb{C}\}$, we consider the algebraic subvariety $\mathcal{X}^{u}$ of $\mathbb{A}^{1} \times \mathbb{P}^{*}\left(V_{\ell}\right)$ obtained as the closure of

$$
\bigcup_{t \in \mathbb{C}^{*}}\{t\} \times \psi_{u}(t) X_{\ell}
$$

in $\mathbb{A}^{1} \times \mathbb{P}^{*}\left(V_{\ell}\right)$, where $\operatorname{SL}\left(V_{\ell}\right)$ acts naturally on the set $\mathbb{P}^{*}\left(V_{\ell}\right)$ of all hyperplanes in $V_{\ell}$ passing through the origin. We now put $\mathcal{L}^{u}:=\operatorname{pr}_{2}^{*} \mathcal{O}_{\mathbb{P}^{*}\left(V_{\ell}\right)}(1)$, where $\operatorname{pr}_{2}: \mathcal{X}^{u} \rightarrow \mathbb{P}^{*}\left(V_{\ell}\right)$ is the restriction to $\mathcal{X}^{u}$ of the projection to the second factor: $\mathbb{A}^{1} \times \mathbb{P}^{*}\left(V_{\ell}\right) \rightarrow \mathbb{P}^{*}\left(V_{\ell}\right)$. The triple

$$
\mu=\left(\mathcal{X}^{u}, \mathcal{L}^{u}, \psi_{u}\right),
$$

is called a test configuration for $(X, L)$ generated by $u$, where we call $\ell$ the exponent of the test configuration $\mu$. If $u=0$, then $\mu$ is called trivial.

For $\mu$ as above, taking the fiber $\mathcal{X}_{0}^{u}$ of $\mathcal{X}^{u}$ over the origin in $\mathbb{A}^{1}$, we consider the Chow weight $q_{\ell}(u)$ for $\mathcal{X}_{0}^{u}$ sitting in $\{0\} \times \mathbb{P}^{*}\left(V_{\ell}\right)\left(\cong \mathbb{P}^{*}\left(V_{\ell}\right)\right)$, i.e., the weight at $\hat{\mathcal{X}}_{0}^{u}$ of the $\mathbb{G}_{m}$-action induced by $\psi_{u}$, where $\hat{\mathcal{X}}_{0}^{u} \in W_{\ell}^{*}$ denotes the Chow form for $\mathcal{X}_{0}^{u}$ viewed as an algebraic cycle on $\mathbb{P}^{*}\left(V_{\ell}\right)$.

Definition 3.1. (1) $\left(X, L^{\otimes \ell}\right)$ is called weakly Chow-stable relative to $T$, if $q_{\ell}(u)<0$ for all $0 \neq u \in\left(\mathfrak{t}_{\ell}^{\perp}\right)_{\mathbb{Z}}$.

(2) $(X, L)$ is called asymptotically weakly Chow-stable relative to $T$, if there exists a positive integer $\ell_{0}$ such that $\left(X, L^{\otimes \ell}\right)$ is weakly Chow-stable relative to $T$ for all positive integers $\ell$ satisfying $\ell \geq \ell_{0}$. 
Remark 3.2. (1) If $\left(X, L^{\otimes \ell}\right)$ is weakly Chow-stable relative to $T$, then by [2], Theorem 3.2, the orbit $T_{\ell}^{\perp} \cdot \hat{X}_{\ell}$ is closed in $W_{\ell}^{*}$.

(2) If $\left(X, L^{\otimes \ell}\right)$ is Chow-stable relative to $T$, then by the Hilbert-Mumford stability criterion, $\left(X, L^{\otimes \ell}\right)$ is weakly Chow-stable relative to $T$.

\section{Strong Relative K-Stability}

For materials in this section, see [5] and [6]. For a maximal algebraic torus $\bar{T}$ in $\operatorname{Aut}(X)$ containing $T$, we fix a Hermitian metric $h$ for $L$ such that $\omega:=c_{1}(L ; h)$ is a Kähler form preserved by the action of the maximal compact subgroup $\bar{T}_{c}$ of $\bar{T}$. Then each $V_{\ell}$ admits a Hermitian structure $\rho_{\ell}$ preserved by the $T_{c}$-action such that

$$
\langle\sigma, \tau\rangle_{\rho_{\ell}}:=\int_{X}(\sigma, \tau)_{h} \omega^{n}, \quad \sigma, \tau \in V_{\ell},
$$

where $(\sigma, \tau)_{h}$ is the pointwise Hermitian pairing of $\sigma$ and $\tau$ on $X$ by the Hermitian metric $h^{\otimes \ell}$. In this section, following [5], we explain how we define the Donaldson-Futaki invariant $F_{1}$ for a sequence of test configurations

$$
\mu_{j}=\left(\mathcal{X}^{u_{j}}, \mathcal{L}^{u_{j}}, \psi_{u_{j}}\right), \quad j=1,2, \ldots,
$$

generated by $u_{j} \in\left(\mathfrak{t}_{\ell_{j}}^{\perp}\right)_{\mathbb{Z}}$, where the positive integer $\ell_{j}$, called the exponent of $\mu_{j}$, is required to satisfy $\ell_{j} \rightarrow+\infty$ as $j \rightarrow \infty$. Let $\mathcal{M}$ be the set of all such sequences $\left\{\mu_{j}\right\}$. For the image $X_{\ell_{j}}$ of $X$ under the Kodaira embedding

$$
\Phi_{\ell_{j}}: X \rightarrow \mathbb{P}^{*}\left(V_{\ell_{j}}\right)
$$

we consider its associated Chow form $\hat{X}_{\ell_{j}} \in W_{\ell_{j}}^{*}:=\left\{\operatorname{Sym}^{d_{\ell_{j}}}\left(V_{\ell_{j}}^{*}\right)\right\}^{\otimes n+1}$. Let $b_{j, \alpha}, \alpha=1,2, \ldots, N_{\ell_{j}}$, be the weights of the $\mathbb{G}_{m}$-action on $V_{\ell_{j}}$ induced by $\psi_{u_{j}}$. We then define the norms $\left\|\mu_{j}\right\|_{1}$ and $\left\|\mu_{j}\right\|_{\infty}$ by

$$
\left\{\begin{array}{l}
\left\|\mu_{j}\right\|_{1}:=\Sigma_{\alpha=1}^{N_{\ell_{j}}}\left|b_{j, \alpha}\right| / \ell_{j}^{n+1}, \\
\left\|\mu_{j}\right\|_{\infty}:=\max \left\{\left|b_{j, 1}\right|,\left|b_{j, 2}\right|, \ldots,\left|b_{j, N_{\ell_{j}}}\right|\right\} / \ell_{j} .
\end{array}\right.
$$

Let $\delta\left(\mu_{j}\right)$ denote $\left\|\mu_{j}\right\|_{\infty} /\left\|\mu_{j}\right\|_{1}$ or 1 according as $\left\|\mu_{j}\right\|_{\infty} \neq 0$ or $\left\|\mu_{j}\right\|_{\infty}=0$. If $\left\|\mu_{j}\right\|_{\infty} \neq 0$, we write $t \in \mathbb{R}_{+}$as $t=\exp \left(s /\left\|\mu_{j}\right\|_{\infty}\right)$ for some $s \in \mathbb{R}$, while we require no relations between $s$ and $t$ if $\left\|\mu_{j}\right\|_{\infty}$ vanishes. Since $\operatorname{SL}\left(V_{\ell_{j}}\right)$ acts naturally on $W_{\ell_{j}}^{*}$, we define a function $f_{u_{j}}=f_{u_{j}}(s)$ in $s$ on $\mathbb{R}$ by

$$
f_{u_{j}}(s):=\delta\left(\mu_{j}\right) \ell_{j}^{-n} \log \left\|\psi_{u_{j}}(t) \cdot \hat{X}_{\ell_{j}}\right\|_{\mathrm{CH}\left(\rho_{\ell_{j}}\right)}, \quad s \in \mathbb{R},
$$


where $W_{\ell_{j}}^{*} \ni w \mapsto\|w\|_{\mathrm{CH}\left(\rho_{\ell_{j}}\right)} \in \mathbb{R}_{\geq 0}$ is the Chow norm for $W_{\ell_{j}}^{*}$ (see [10]). Taking the derivative $\dot{f}_{u_{j}}(s):=d f_{u_{j}} / d s$, we define $F_{1}\left(\left\{\mu_{j}\right\}\right) \in \mathbb{R} \cup\{-\infty\}$ by

$$
F_{1}\left(\left\{\mu_{j}\right\}\right):=\lim _{s \rightarrow-\infty}\left\{\varliminf_{j \rightarrow \infty} \dot{f}_{u_{j}}(s)\right\} .
$$

Definition 4.2 (cf. [6]). (1) $(X, L)$ is called strongly $K$-semistable relative to $T$, if $F_{1}\left(\left\{\mu_{j}\right\}\right) \leq 0$ for all $\left\{\mu_{j}\right\} \in \mathcal{M}$.

(2) Let $(X, L)$ be strongly $\mathrm{K}$-semistable relative to $T$. Then $(X, L)$ is called strongly $K$-stable relative to $T$, if for every $\left\{\mu_{j}\right\} \in \mathcal{M}$ satisfying $F_{1}\left(\left\{\mu_{j}\right\}\right)=$ 0 , there exists a $j_{0}$ such that for all $j \geq j_{0}, \mu_{j}$ is trivial, i.e., $u_{j}=0$.

Note that neither strong K-semistability relative to $T$ nor strong Kstability relative to $T$ depends on the choice of $\bar{T}$ and $h$ (see 8$]$ ).

\section{Proof of Main Theorem}

In this section, we consider a polarized algebraic manifold $(X, L)$ which is strongly K-stable relative to $T$. The proof is divided into two parts.

Step 1 . We shall first show that $(X, L)$ is asymptotically weakly Chow-stable relative to $T$. Assume the contrary for contradiction. Then we can find an increasing sequence of positive integer $\ell_{j}, j=1,2, \ldots$, such that

$$
\ell_{j} \rightarrow+\infty, \quad \text { as } j \rightarrow \infty,
$$

and that $\left(X, L^{\otimes \ell_{j}}\right)$ is not weakly Chow-stable relative to $T$ for any $j$. Then by Definition 3.1, to each $j$, we can assign a element $0 \neq u_{j} \in\left(\mathfrak{t}_{\ell_{j}}^{\perp}\right)_{\mathbb{Z}}$ such that $q_{\ell_{j}}\left(u_{j}\right) \geq 0$. Recall that (see for instance 4], Appendix I)

$$
q_{\ell_{j}}\left(u_{j}\right)=\left\|\mu_{j}\right\|_{1} \ell_{j}^{n} \lim _{s \rightarrow-\infty} \dot{f}_{u_{j}}(s) .
$$

Since the function $\dot{f}_{u_{j}}(s)$ is non-decreasing in $s$ for each $j$, it follows that

$$
0 \leq\left\|\mu_{j}\right\|_{1}^{-1} \ell_{j}^{-n} q_{\ell_{j}}\left(u_{j}\right) \leq \dot{f}_{u_{j}}(s), \quad-\infty<s<+\infty .
$$

Hence $0 \leq \dot{f}_{u_{j}}(s)$ for each fixed $s \in \mathbb{R}$. Taking $\underline{\lim }$ as $j \rightarrow \infty$, we have

$$
0 \leq \varliminf_{j \rightarrow \infty} \dot{f}_{u_{j}}(s),
$$

for every $s \in \mathbb{R}$. By taking limit of (5.1) as $s \rightarrow-\infty$, we obtain

$$
0 \leq \lim _{s \rightarrow-\infty} \varliminf_{j \rightarrow \infty} \dot{f}_{u_{j}}(s)=F_{1}\left(\left\{\mu_{j}\right\}\right) .
$$

Since $(X, L)$ is strongly $\mathrm{K}$-stable relative to $T$, this inequality implies that $F_{1}\left(\left\{\mu_{j}\right\}\right)$ vanishes. Again by strong K-stability of $(X, L)$ relative to $T$, there 
exists a $j_{0}$ such that $\mu_{j}$ are trivial for all $j$ with $j \geq j_{0}$ in contradiction to $u_{j} \neq 0$, as required.

Step 2. In view of (1) of Remark 3.2, we see from Step 1 above that the orbit $O_{\ell}:=T_{\ell}^{\perp} \cdot \hat{X}_{\ell}$ is closed in $W_{\ell}^{*}$. Hence $O_{\ell}$ is an affine algebraic subset of $W_{\ell}^{*}$. Since $O_{\ell}$ is closed in $W_{\ell}^{*}$, we here observe that:

$$
O_{\ell} \cap \mathbb{C} \hat{X}_{\ell} \text { is a finite set, }
$$

where $\mathbb{C} \hat{X}_{\ell}$ is the one-dimensional vector subspace of $W_{\ell}^{*}$ generated by $\hat{X}_{\ell}$. Consider the identity components $H_{\ell}$ and $H_{\ell}^{\prime}$ of the isotropy subgroups of the reductive algebraic group $T_{\ell}^{\perp}$ at the point $\hat{X}_{\ell}$ and $\left[\hat{X}_{\ell}\right]$, respectively. Since $\operatorname{dim} H_{\ell}=\operatorname{dim} H_{\ell}^{\prime}$ by (5.2), it suffices to show that an $\ell_{0}$ exists such that $\operatorname{dim} H_{\ell}=0$ for all $\ell$ with $\ell \geq \ell_{0}$. Assume the contrary for contradiction. Then we have an increasing sequence of positive integers $\ell_{j}$ such that

$$
\operatorname{dim} H_{\ell_{j}}>0, \quad j=1,2, \ldots,
$$

and that $\ell_{j} \rightarrow+\infty$, as $j \rightarrow \infty$. Since by [7] the isotropy subgroup $H_{\ell_{j}}$ of the reductive algebraic group $T_{\ell_{j}}^{\perp}$ at the point $\hat{X}_{\ell_{j}}$ is a reductive algebraic group, the group $H_{\ell_{j}}$ contains a nontrivial algebraic torus $\mathbb{G}_{m}$. Since (5.2) allows us to obtain a natural isogeny $\iota: H_{\ell_{j}} \rightarrow \bar{H}_{\ell_{j}}$ from $H_{\ell_{j}}$ to an algebraic subgroup $\bar{H}_{\ell_{j}}$ of $\operatorname{Aut}(X)$, the image $\bar{H}_{\ell_{j}}$ also contains a nontrivial algebraic torus $G_{j}=\mathbb{G}_{m}$. For $\bar{T}$ in Section 4 , replacing $\bar{T}$ by its conjugate in $\operatorname{Aut}(X)$ if necessary, we may assume that $\bar{T}$ contains $G_{j}$. For the maximal compact subgroup $\left(G_{j}\right)_{c}$ of $G_{j}$, we choose a generator $u_{j} \neq 0$ for the one-dimensional real Lie subalgebra

$$
\sqrt{-1}\left(\mathfrak{g}_{j}\right)_{c}:=\sqrt{-1} \operatorname{Lie}\left(\left(G_{j}\right)_{c}\right)
$$

in $\mathfrak{t}_{\ell_{j}}^{\perp} \cap H^{0}\left(X, \mathcal{O}\left(T_{X}\right)\right)$ such that $\exp \left(2 \pi \sqrt{-1} u_{j}\right)=\mathrm{id}_{X}$. Then for the algebraic group homomorphisms $\psi_{u_{j}}: \mathbb{G}_{m} \rightarrow T_{\ell}^{\perp} \subset \mathrm{SL}\left(V_{\ell_{j}}\right)$ generated by $u_{j}$, we obtain the associated test configurations

$$
\mu_{u_{j}}=\left(\mathcal{X}^{u_{j}}, \mathcal{L}^{u_{j}}, \psi_{u_{j}}\right), \quad j=1,2, \ldots,
$$

for $(X, L)$ generated by $u_{j}$. Let $\beta_{j}$ be the weight of the $\mathbb{G}_{m}$-action by $\psi_{u_{j}}$ at $\hat{X}_{\ell_{j}}$. Since $\psi_{u_{j}}(t) \cdot \hat{X}_{\ell_{j}}=t^{\beta_{j}} \hat{X}_{\ell_{j}}$, by differentiating the functions $f_{u_{j}}(s)$ in (4.1) with respect to $s$, we obtain

$$
\dot{f}_{u_{j}}(s)=\ell_{j}^{-n} \beta_{j} /\left\|\mu_{u_{j}}\right\|_{1}, \quad-\infty<s<+\infty .
$$

Replacing $u_{j}$ by $v_{j}:=-u_{j}$, we also have the test configurations

$$
\mu_{v_{j}}=\left(\mathcal{X}^{v_{j}}, \mathcal{L}^{v_{j}}, \psi_{v_{j}}\right), \quad j=1,2, \ldots,
$$


for $(X, L)$ generated by $v_{j}$. Replace $u_{j}$ by $v_{j}$ in (4.1). Then by differentiating the functions $f_{v_{j}}(s)$ with respect to $s$, we obtain

$$
\dot{f}_{v_{j}}(s)=-\ell_{j}^{-n} \beta_{j} /\left\|\mu_{v_{j}}\right\|_{1}, \quad-\infty<s<+\infty .
$$

Note that $\left\|\mu_{u_{j}}\right\|_{1}=\left\|\mu_{v_{j}}\right\|_{1}$. The right-hand side of (5.3) and the righthand side of (5.4) are both bounded from above by a positive constant independent of $j$ (see [5], Section 3). Hence, replacing $\left\{u_{j} ; j=1,2, \ldots\right\}$ by its subsequence if necessary, we may assume that

$$
\left\{\ell_{j}^{-n} \beta_{j} /\left\|\mu_{u_{j}}\right\|_{1} ; j=1,2, \ldots,\right\}
$$

is a convergent sequence. Let $\gamma$ be its limit. Then by (5.3) and (5.4), $F_{1}\left(\left\{\mu_{u_{j}}\right\}\right)=\gamma=-F_{1}\left(\left\{\mu_{v_{j}}\right\}\right)$. Since $(X, L)$ is strongly K-stable relative to $T$, the inequalities $F_{1}\left(\left\{\mu_{u_{j}}\right\}\right) \leq 0$ and $F_{1}\left(\left\{\mu_{v_{j}}\right\}\right) \leq 0$ hold, and hence

$$
\gamma=0
$$

Again by strong K-stability of $(X, L)$ relative to $T$, we see that $\mu_{u_{j}}$ are trivial for $j \gg 1$, so that $u_{j}=0$ for $j \gg 1$ in contradiction, as required.

\section{REFERENCES}

[1] S.K. Donaldson: Scalar curvature and stability of toric varieties, J. Differential Geom. 62 (2002), 289-349.

[2] T. Mabuchi Stability of extremal Kähler manifolds Osaka J. Math. 41 (2004), $563-582$.

[3] T. MABUChI Asymptotics of polybalanced metrics under relative stability constraints, Osaka J. Math. 48 (2011), 845-856.

[4] T. Mabuchi: Relative stability and extremal metrics, to appear in J. Math. Soc. Japan.

[5] T. Maвuchi: The Donaldson-Futaki invariant for sequences of test configurations, arXiv: 1307.1957.

[6] T. MABUCHI: Existence problem of extremal Kähler metrics, in preparation.

[7] Y. Matsushima: Espaces homogènes de Stein des groupes de Lie complexes, Nagoya Math. J. 18 (1961), 153-164.

[8] T. Mabuchi and Y. NitTa: Completion of the space of test configurations, in preparation.

[9] G. SzÉKelyhidi: Extremal metrics and K-stability, Bull. London Math. Soc. 39 (2007), 76-84.

[10] S. Zhang: Heights and reductions of semi-stable varieties, Compositio Math. 104 (1996), 77-105.

Department of Mathematics, Graduate School of Science, Osaka UniverSITY, TOYONAKA, OsAKA, 560-0043 JAPAN, mabuchi@math.sci.osaka-u.ac.jp 
Department of Mathematics, Graduate School of Science, Tokyo Institute of Technology, Megro, Tokyo, 152-8551 Japan, n_yakkun82@yahoo.co.jp 